\title{
BMJ Open Global epidemiology and patterns of cerebral venous thrombosis: a systematic review and meta-analysis protocol
}

\author{
Celestin Danwang, ${ }^{1}$ Temgoua Ngou Mazou, ${ }^{2}$ Joël Noutakdie Tochie, ${ }^{1}$ \\ Ronni Tankeu, ${ }^{2}$ Jean Joel Bigna ${ }^{3,4}$
}

To cite: Danwang C, Mazou TN, Tochie JN, et al. Global epidemiology and patterns of cerebral venous thrombosis: a systematic review and metaanalysis protocol. BMJ Open 2018;8:e19939. doi:10.1136/ bmjopen-2017-019939

- Prepublication history and additional material for this paper are available online. To view these files, please visit the journal online (http://dx.doi. org/10.1136/bmjopen-2017019939).

Received 4 0ctober 2017 Revised 28 February 2018 Accepted 6 March 2018

Check for updates

${ }^{1}$ Department of Surgery and Specialties, Faculty of Medicine and Biomedical Sciences,

University of Yaoundé I, Yaoundé, Cameroon

${ }^{2}$ Department of Internal Medicine and Specialties, Faculty of Medicine and Biomedical Sciences, University of Yaoundé I, Yaoundé, Cameroon

${ }^{4}$ Department of Epidemiology and Public Health, Centre Pasteur of Cameroon, Yaoundé, Cameroon

${ }^{3}$ School of Public Health, Faculty of Medicine, University of ParisSud XI, Le Kremlin-Bicêtre, France

Correspondence to Dr Celestin Danwang; danram07@yahoo.fr

\section{ABSTRACT}

Introduction Venous thrombosis can affect all veins in the body including cerebral veins, where it causes cerebral venous thrombosis (CVT). CVT is an aetiology of stroke, particularly in children and young adults. Its clinical features vary widely according to the occluded vessel. Data concerning the epidemiology of CVT are scant. This protocol for a systematic review and meta-analysis aims to critically synthesise data concerning prevalence, incidence, risk factors, anatomical patterns, diagnostic and therapeutic delays, and mortality rate of CVT in the global population.

Methods and analysis MEDLINE, EMBASE and ISI

Web of Science databases will be searched for relevant abstracts of studies published between 1 January 1990 and 31 0ctober 2017, without language restriction. After the screening of abstracts, study selection, data extraction and assessment of risk of bias, we will assess studies individually for heterogeneity. Random effects metaanalysis will then be used to pool studies judged to be clinically homogenous. Funnel plot analysis and Egger's test will be used to detect publication bias. Results will be presented according to economic level of the various countries (high-income vs low/middle-income countries). Ethics and dissemination Since the current study will be based on published data, ethical approval is not required. This review is expected to provide relevant data to help in evaluating the global burden of CVT. The final report of this study will be published in a peer-reviewed journal. PROSPERO registration number CRD42017074266.

\section{INTRODUCTION}

Venous thrombosis can affect all veins in the body including the cerebral venous system leading to cerebral venous thrombosis (CVT). ${ }^{1}$ Although rare and affecting all age groups, CVT is a potential aetiology of stroke, particularly in children and young adults. ${ }^{23}$ CVT accounts for $0.5 \%$ of all strokes, and its annual incidence ranges from three to four cases per million among the general population up to seven cases per million among the youths, ${ }^{2} 3$ with young adult women being more affected than men. The established risk factors for CVT are ear, nose and facial infections as well as intracranial tumours, pregnancy and the puerperium, systemic diseases, coagulopathies, oral
Strengths and limitations of this study

- A limitation to the current review may be the scant amount of data; mainly case series done in urban settings which may pose a major restriction to this study, as the result may not reflect the true burden of cerebral venous thrombosis (CVT).

- To the best of our knowledge, this is the first review to summarise available data on the global burden of CVT.

- The current review will include studies without language restrictions, and thus will allow to enrol the maximum of studies published on the topic.

contraceptives use and dehydration. ${ }^{4}$ The clinical presentation varies widely according to the occluded cerebral vessel. ${ }^{3}$

The recent advances in neuroimaging techniques with the widespread use of MRI even in low/middle-income countries (LMIC) have made the diagnosis of CVT easier, hence improving on the overall prognosis of patients with CVT. ${ }^{2}$ The various therapeutic options are anticoagulation therapy (mainly with heparin), mechanical thrombectomy, intravenous thrombolytic and decompressive hemicraniectomy. ${ }^{2}$

The current epidemiological data on CVT are derived from primary studies in which all ethnicities are often not represented making it impossible to appraise the global scene. ${ }^{56}$ Despite this gap in knowledge on the subject matter, till date, no study has focused on the global epidemiology, and the risk factors for CVT in all racial backgrounds and all countries of various income levels. ${ }^{7}$ Accordingly, we propose this protocol for a systematic review and meta-analysis to critically synthesise contemporary evidence on the occurrence of CVT in the world. The overall research goal is to provide useful data for health authorities.

\section{OBJECTIVE}

This systematic review and meta-analysis aims at determining the prevalence, incidence, risk 
factors, anatomical patterns, diagnostic and therapeutic delays and mortality rate of CVT on a global basis.

\section{REVIEW QUESTIONS}

Specifically, the proposed systematic review will answer the following questions:

1. What are the global prevalence and incidence of CVT?

2. What are the risk factors of CVT?

3. What are the neuroimaging features (the various anatomical locations) of CVT?

4. What is the delay between the onset of symptoms, the diagnosis and initiation of treatment according to the country's income level (LMICs vs high-income countries)?

5. What is the global case fatality rate due to CVT?

\section{METHODS AND ANALYSIS}

This systematic review and meta-analysis will be reported in conformity with Meta-analysis of Observational Studies in Epidemiology guidelines. ${ }^{8}$ For the present protocol, the Preferred Reporting Items for Systematic Review and Meta-Analysis Protocols (PRISMA-P) was used for the reporting. ${ }^{9}$ Online supplementary file 1 shows the PRISMA checklist used for this study protocol.

\section{Criteria for considering studies for the review Inclusion criteria}

1. We intend to include cross-sectional studies, casecontrol, cohort studies and case series with at least 30 participants.

2. All aforementioned studies published between 1 January 1990 and 31 October 2017 without any language restriction.

3. Observational studies with sufficient data on the prevalence and/or incidence, risk factors, anatomical patterns, diagnostic and therapeutic delays and mortality rate of CVT.

\section{Exclusion criteria}

We will exclude:

1. Commentaries, editorials, letters and reviews.

2. Studies with inaccessible full texts either online or from the corresponding author.

3. Studies in which relevant data on CVT are impossible to extract.

4. For duplicates or studies published in more than one report, the study reporting the largest sample size will be considered.

\section{Search strategy for identifying relevant studies}

The search strategy will be as follows:

\section{Bibliographic database searches}

1. Excerpta Medica Database (EMBASE), MEDLINE through PubMed and Web of Science (Science Citation Index) databases will be searched for relevant studies concerning CVT from 1 January 1990 to

\begin{tabular}{ll}
\hline Table 1 & Search strategy for PubMed \\
\hline $\begin{array}{l}\text { Search } \\
\text { number }\end{array}$ & Search terms \\
\hline 1 & $\begin{array}{l}\text { Cerebral Venous Thrombosis OR Cerebral Vein } \\
\text { Thrombosis OR Thrombosis of the cerebral vein } \\
\text { and sinus OR Cavernous sinus thrombosis }\end{array}$ \\
\hline 2 & $\begin{array}{l}\text { Limits: } 1 \text { January } 1990 \text { to 31 October 2017; no } \\
\text { language restriction }\end{array}$ \\
\hline 3 & 1 AND 2 \\
\hline
\end{tabular}

31 October 2017, with no language restriction. The research strategy will be designed for MEDLINE and adapted for other databases using both text words and medical subject heading terms related to CVT (table 1).

2. The abstracts of all eligible articles will be reviewed and full-text articles will be accessed through PubMed, EMBASE, Scopus Database, AJOL, Google Scholar, HINARI or journals' websites. The authors of papers whose full texts will not be obtained by internet-based sources will be directly contacted to provide them.

\section{Searching for other sources}

The references cited by identified studies will also be searched for additional data source.

\section{Selection of studies for inclusion in the review}

The titles and abstracts of papers obtained from the search will be independently scrutinised by two reviewers $(\mathrm{CD}$ and TNM) using an assessment guide. The full texts of potentially eligible papers will be retrieved by one reviewer (CD). Thereafter, they will independently review the full text of each potentially eligible study, compare their results and resolve any discrepancy by discussion. If an agreement is not reached after discussion, a third reviewer (JNT) will be consulted for arbitration.

\section{Assessment of methodological quality and reporting of data}

An adapted version of the Risk of Bias Tool for Prevalence Studies developed by Hoy et $a l^{10}$ (see online supplementary file 2) will be used to evaluate included studies for the quality and risk of bias, and will be applied to screened fulltext articles by two reviewers.

\section{Data extraction and management}

A data extraction form (see online supplementary file 3) will be used by two independent reviewers (CD and TNM) to collect information on the last name of the first author, year of publication, region (Africa, Americas, Europe, Asia, Oceania), country economic level (high-income countries vs LMICs), country, study design, study area (rural vs urban), study setting (intensive care unit, surgery unit, post mortem), sample size, mean or median age, age range, male proportion, specific characteristics of the study population (women on oral contraceptives, patients with HIV, pregnant/postpartum women and postoperative patients), prevalence rate, incidence rate, anatomical patterns (occluded cerebral vessel 
on neuroimaging studies), diagnostic and therapeutic delays (days or weeks), and/or mortality rate of CVT and risk factors for CVT in the study population. For multinational studies, the prevalence, incidence or mortality will be reported for the individual countries.

\section{Data synthesis and analysis}

When data collection will be complete, a meta-analysis will be conducted. The study-specific estimates will be pooled by using a random effects meta-analysis model to obtain an overall summary estimate of the prevalence and/or incidence across studies, after stabilising the variance of individual studies with the use of the Freeman-Tukey double arcsine transformation. ${ }^{11}$ SEs for the study-specific estimates will be determined from the point estimate and the appropriate denominators. Heterogeneity will be assessed using the $\chi^{2}$ test on Cochrane's $\mathrm{Q}$ statistic ${ }^{12}$ which is quantified by $\mathrm{I}^{2}$ values, assuming that $\mathrm{I}^{2}$ values of $25 \%, 50 \%$ and $75 \%$, respectively, represent low, medium and high heterogeneity. ${ }^{13}$ We will assess the presence of publication bias using funnel plots and Egger's test. ${ }^{14}$ Publication bias will be confirmed if $\mathrm{p}$ value on Egger's test is $<0.10$. Where substantial heterogeneity will be detected, subgroup and meta-regression analyses will be performed to investigate the possible sources of heterogeneity using previously mentioned variables and the study methodological quality. In case of substantial clinical heterogeneity, a narrative summary of findings will be done. The inter-rater agreement for study inclusion will be assessed using Cohen's $\kappa$ coefficient. ${ }^{15}$ Data analyses will use the 'meta' package of the statistical software R (V.3.4.4 (15 March 2018), the R Foundation for statistical computing, Vienna, Austria).

\section{Presentation and reporting of results}

The study selection process will be summarised in a flow diagram. Quantitative data will be presented in evidence tables of individual studies as well as in summary tables and forest plots where appropriate. The quality scores and risk of bias for each eligible study will be reported accordingly. This may be tabulated and accompanied by narrative summaries.

\section{Patient and public involvement}

In this study, data will not be collected directly from patients, but in published studies available in main databases.

\section{CONCLUSION}

Thrombosis of the cerebral veins and sinus represents one of the causes of stroke, especially in children and young adults. With the current scarcity of global representative epidemiological data on CVT, the findings of this systematic review may help inform policymakers on the global burden of this deadly disease.

\section{Ethics and disseminations}

The current review will use published studies. Therefore, there is no requirement for ethical approval. The review is expected to provide the current global burden of CVT in order to inform health authorities and decision makers to elaborate effective preventive strategies to reduce the burden of CVT. The resulting manuscript will be published in a peer-reviewed journal.

Contributors CD, TNM and JNT had the idea. CD, JNT and JJB designed and conceived the protocol. CD made the first draft. CD, TNM, JNT, RT and JJB critically revised the methodology and intellectual content. CD and JJB are the guarantors of the review. All authors approved the final version of this manuscript.

Funding This research received no specific grant from any funding agency in the public, commercial or not-for-profit sectors.

Competing interests None declared.

Patient consent Not required.

Provenance and peer review Not commissioned; externally peer reviewed.

Open Access This is an Open Access article distributed in accordance with the Creative Commons Attribution Non Commercial (CC BY-NC 4.0) license, which permits others to distribute, remix, adapt, build upon this work non-commercially, and license their derivative works on different terms, provided the original work is properly cited and the use is non-commercial. See: http://creativecommons.org/ licenses/by-nc/4.0/

(C) Article author(s) (or their employer(s) unless otherwise stated in the text of the article) 2018. All rights reserved. No commercial use is permitted unless otherwise expressly granted.

\section{REFERENCES}

1. Heit JA. Epidemiology of venous thromboembolism. Nat Rev Cardiol 2015;12:464-74.

2. Bousser MG, Ferro JM. Cerebral venous thrombosis: an update. Lancet Neurol 2007;6:162-70.

3. Stam J. Thrombosis of the cerebral veins and sinuses. N Engl J Med 2005;352:1791-8.

4. Saadatnia M, Fatehi F, Basiri K, et al. Cerebral venous sinus thrombosis risk factors. Int J Stroke 2009;4:111-23.

5. Coutinho JM, Zuurbier SM, Aramideh M, et al. The incidence of cerebral venous thrombosis: a cross-sectional study. Stroke 2012;43:3375-7.

6. Pillai L, Ambike D, Nirhale S, et al. Cerebral venous thrombosis: an experience with anticoagulation with low molecular weight heparin. Indian J Crit Care Med 2005;9:14-18.

7. Sidhom Y, Mansour M, Messelmani M, et al. Cerebral venous thrombosis: clinical features, risk factors, and long-term outcome in a Tunisian cohort. J Stroke Cerebrovasc Dis 2014;23:1291-5.

8. Stroup DF, Berlin JA, Morton SC, et al. Meta-analysis of observational studies in epidemiology: a proposal for reporting. Meta-analysis Of Observational Studies in Epidemiology (MOOSE) group. JAMA 2000;283:2008-12.

9. Moher D, Shamseer L, Clarke M, et al. Preferred reporting items for systematic review and meta-analysis protocols (PRISMA-P) 2015 statement. Syst Rev 2015;4:1.

10. Hoy $D$, Brooks $P$, Woolf $A$, et al. Assessing risk of bias in prevalence studies: modification of an existing tool and evidence of interrater agreement. J Clin Epidemiol 2012;65:934-9.

11. Miller JJ. The inverse of the freeman - tukey double arcsine transformation. The American Statistician 1978;32:138.

12. Kulinskaya E, Dollinger MB. An accurate test for homogeneity of odds ratios based on Cochran's Q-statistic. BMC Med Res Methodol 2015;15:49.

13. Huedo-Medina TB, Sánchez-Meca J, Marín-Martínez F, et al. Assessing heterogeneity in meta-analysis: Q statistic or 12 index? Psychol Methods 2006;11:193-206.

14. Egger M, Davey Smith G, Schneider M, et al. Bias in meta-analysis detected by a simple, graphical test. BMJ 1997;315:629-34.

15. McHugh ML. Interrater reliability: the kappa statistic. Biochem Med 2012;22:276-82. 